\section{Awareness of fitness to drive among cardiologists in Saudi Arabia}

Rami M. Abazid, FSCCT, MD, Ahmad Almeman, MD, PhD,

Haitham Sakr, FRCP, Akram F. Eldesoky, MD,

Hisham M. Eissa, MSc, Tawfeeq H. Alharbi, MD,

Abdullah A. Altorbag, MSc, Osama A. Smettei, FRCP, MD

\section{ABSTRACT}

Objectives: To assess the perception and awareness of cardiologists in Saudi Arabia about medical fitness to drive in different cardiovascular diseases.

Methods: The study is a cross-sectional survey-based study between June 2018 and July 2018. Cardiologists were asked to complete a self-administered questionnaire inquiring about awareness of driving fitness and educating patients regarding driving risks in specific cardiovascular conditions.

Results: A total of 194 cardiologists completed the study survey; there were $30.4 \%$ consultants, $59.3 \%$ specialists, and $10.3 \%$ residents. Out of $195,72 \%$ were aware of the existence of particular international driving regulations for cardiovascular diseases, whereas 28\% were not aware. Although, no Saudi guidelines assessing fitness-to-drive are available, $11 \%$ of the participants claimed awareness of such regulations. Interestingly, we found that cardiologists had never or rarely educated their patients regarding the potential risks of driving: $49 \%$ in symptomatic angina, $47 \%$ when ejection fraction is $\leq 35 \%, 39 \%$ in symptomatic valvular diseases, $26 \%$ after cardioverter defibrillators implantation, and $23 \%$ after nonelective percutaneous coronary interventions.

Conclusion: There is a lack of awareness among cardiologists in Saudi Arabia about international guidelines regarding medical driving fitness. This study highlights the necessity of formulating appropriate national driving regulations for cardiovascular diseases.

Saudi Med J 2019; Vol. 40 (1): 93-96 doi: 10.15537/smj.2019.1.23595

$\mathrm{R}$ oad traffic accidents are among the leading causes of death in Saudi Arabia. ${ }^{1}$ To be performed safely, driving requires proper responses including visual, motor, and cognitive skills, as stated in Driver's and Vehicle License Agency (DVLA) reports. ${ }^{2}$ In fact, multiple medical disorders can result in either mental or physical disabilities, which can have an acute or chronic course. Therefore, fitness to drive should be evaluated in all medical conditions that may affect an individual's ability to drive safely. Moreover, chronic illnesses should be periodically and consistently evaluated in an appropriate manner for the possibility of any further deterioration or improvement based on the current clinical status that would change the previous driving fitness decision. ${ }^{2}$ Cardiovascular-related symptoms, especially syncope or dizziness have significant consequences for vehicle driving. ${ }^{3}$ Subsequently, a proper driving fitness determination in heart diseases is vitally important to maintain the patient's and the public's safety. ${ }^{4}$ The following elements are strictly required to be intact while driving: vision, perception, hearing, attention, memory, judgment, adaptive strategy, planning, coordination, muscle power, and strength. These elements are affected by several diseases, including cardiovascular, renal, endocrine, neurological, psychiatric, and other conditions, which may lead to fatal consequences. ${ }^{2}$ Some driving agencies, such as the DVLA in Britain and Austroads in Australia, have set particular standards for some diseases where each diseases is well characterized in terms of the ability of patients to drive safely. ${ }^{2,5}$ All provide clear outlines for clinicians regarding the capabilities of drivers based on the best available medical evidence. Importantly, cardiovascular diseases (CVD) have an important effect on driving capability and safety. In fact, acquired and congenital heart diseases are classified into 30 different categories, such as angina, hypertension, acute coronary syndrome, aortic aneurysm, arrhythmias, and so on. ${ }^{2}$ Based on their characteristics, some of these are considered to be contraindication for vehicle driving, either permanently or for a limited time. Accordingly, driver's licenses for some patients with serious heart diseases should be restricted. ${ }^{2}$ Several studies have shown that patients with cardiovascular disease are at a higher risk for car accidents, which puts them, their families, and the public in danger. ${ }^{6}$ When assessing patients with CVD, cardiologists should be trained to properly evaluate the associated incapacitation that may affect driving safety. Making such an assessment is uncomfortable, as it may limit the patient's ability to enjoy life, which may put $\mathrm{him} /$ her at higher risk of anxiety or depression. On the other hand, it may help to save the patient's life. ${ }^{7}$

Disclosure. Authors have no conflict of interests, and the work was not supported or funded by any drug company. 
The aim of this study is to evaluate awareness of fitness to drive among cardiologists.

Methods. This was an observational cross-sectional descriptive study based on an online survey. All questions were extracted from the DVLA, ${ }^{1}$ a set of fitness to drive guidelines in Britain. Ethical approval was obtained from the Regional and Institutional Ethical Committee. The participants were all cardiologists practicing in Saudi Arabia (KSA). We chose several governmental and private hospitals in the central region of Saudi Arabia (Al Qassim, Riyadh, and Hail). The hospitals were selected using a computer system and was based on a regionally restricted cluster randomization strategy. Due to the difficulty of obtaining cardiologists at random inside the selected hospitals, we used a convenience sampling method. After obtaining consent, all eligible physicians were invited to participate in the survey. We only excluded cardiologists who refused to participate.

The survey questions were obtained from a well validated guideline (DVLA), and the original English language of the survey was used. Hence, survey validation was not required. Nonetheless, the construction of the questions was subjected to several phases. Phase I: identifying the risk factors from the guidelines. ${ }^{2}$ Phase II: selecting the questions by experts to detect the causality that resulted from driving. Phase III: pilot testing among groups of experts for content validity. Phase IV: pilot testing among groups of participants for content validity. Phase V: reliability testing using Cronbach's alpha coefficient.

The cardiologists answered an online questions sheet regarding their basic demographics. As there are no clear driving guidelines for cardiac patients in KSA the questions were simplified to ease the process of understanding. The survey was consisting of 15 multiple choice questions and divided into sections based on the clinical diagnosis (coronary artery disease, recent revascularization, cardiomyopathy, arrhythmias, and device implantation); and echocardiography (ejection fraction and valvular heart disease). A Likert scale was used in the response section. Each subsection contained specific cases on when a cardiac patient should or should not drive, based on the guidelines. The cardiologists were asked to answer all of them based on their current practices and knowledge (Table 1).

Statistical analysis. Descriptive statistics were performed for demographics, experience, physician position, and other data were reported as percentages. Kruskal-Wallis test was used to compare the categorical variable among different groups, $p<0.05$ was considered statistically significant. All statistical analyses were performed using Statistical Package for the Social Sciences for Windows, Version 19.0 (SPSS Inc., Chicago, IL, USA).

Results. There were 206 participants in our study; after excluding 4 non-cardiologists and 8 with incomplete data, the final number of participants in the survey was 194 cardiologists, among whom there were $20(10.3 \%)$ residents, $115(59.3 \%)$ specialists, and 59 (30.4\%) consultants. The mean age was $47 \pm 7$ years, men were 174 (89\%). Eighty-four (43\%) had more than 10 years of experience, 64 (33\%) had 5-10 years of experience, and $46(24 \%)$ had less than 5 years of experience. We found that $140(72 \%)$ were aware of the existence of particular international driving regulations for cardiovascular diseases, whereas 54 (28\%) were not aware. Although, no Saudi guidelines assessing fitnessto-drive are available, $22(11 \%)$ of the study participants claimed awareness of such regulations.

Table 1 - Attitudes of cardiologists toward assessing fitness to drive for different cardiovascular diseases.

\begin{tabular}{lccccc}
\hline Diagnosis & $\begin{array}{c}\text { Always } \\
\mathbf{n}(\%)\end{array}$ & $\begin{array}{c}\text { Often } \\
\mathbf{n}(\%)\end{array}$ & $\begin{array}{c}\text { Sometimes } \\
\mathbf{n}(\%)\end{array}$ & $\begin{array}{c}\text { Rarely } \\
\mathbf{n}(\%)\end{array}$ & $\begin{array}{c}\text { Never } \\
\mathbf{n}(\%)\end{array}$ \\
\hline Symptomatic stable angina & $25(13.0)$ & $21(11.0)$ & $52(27.0)$ & $45(23.0)$ & $51(26.0)$ \\
Symptomatic valvular diseases & $33(17.0)$ & $26(13.4)$ & $60(31.0)$ & $35(18.0)$ & $40(20.6)$ \\
Symptomatic tachyarrhythmia & $74(38.0)$ & $37(19.0)$ & $53(27.3)$ & $13(6.7)$ & $17(9.0)$ \\
Symptomatic bradyarrhythmia & $88(45.4)$ & $44(22.7)$ & $36(18.5)$ & $12(6.2)$ & $14(7.2)$ \\
After CABG & $57(29.5)$ & $27(13.9)$ & $49(25.2)$ & $27(13.9)$ & $34(17.5)$ \\
After PCI or ACS & $66(34.4)$ & $38(19.5)$ & $45(23.0)$ & $23(11.8)$ & $22(11.3)$ \\
After ICD implantation & $57(29.4)$ & $41(21.2)$ & $45(23.0)$ & $25(13.0)$ & $26(13.4)$ \\
Well functioning PPM & $16(8.3)$ & $16(8.3)$ & $32(16.4)$ & $44(22.7)$ & $86(44.3)$ \\
In patients with EF <35\% & $14(7.2)$ & $20(10.5)$ & $68(35.0)$ & $38(19.5)$ & $54(27.8)$ \\
\hline \multicolumn{2}{c}{ CABG - coronary artery bypass graft , PCI - percutaneous coronary intervention, ACS - acute coronary } \\
syndrome, PPM - permanent pacemakers, EF - ejection traction, ICD - cardioverter defibrillators implantation \\
\hline
\end{tabular}


Interestingly, we found that cardiologists had never or rarely educated their patients regarding the potential risks of driving: $96(49 \%)$ in symptomatic angina, $92(47 \%)$ when ejection fraction is $\leq 35 \%, 75$ $(39 \%)$ in symptomatic valvular diseases, 51 (26\%) after cardioverter defibrillators implantation, 45 (23\%) after non-elective percutaneous coronary interventions, and $35 \%$ in overall CVD. The overall percentages and frequencies of the attitudes of cardiologists toward assessing fitness to drive for different cardiovascular diseases are shown in Table 1 and Figure 1. The attitudes of cardiologists toward assessing fitness to drive for different cardiovascular diseases are shown in Table 1.

The lack of awareness regarding driving fitness did not differ significantly among residents $(6 / 20 ; 30 \%)$, specialists $(31 / 115 ; 27 \%)$, and consultants (17/59; $29 \%)(p=0.9)$. Similar results were found among cardiologists with experience less than 5 years (17/46; $37 \%)$, 5-10 years $(15 / 64 ; 23.5 \%)$, and more than 10 years $(22 / 84 ; 26 \%)(p=0.4)$.

Discussion. The study was conducted to evaluate cardiologists' awareness of the recommendations for restrictions to vehicle driving in the most common CVD. Importantly we found that $28 \%$ of cardiologists were not aware of any medical-related driving guidelines. Generally, the results of this study indicate that further steps are essential to increase the awareness of cardiologists and further educate them on the international guidelines. Surprisingly, a few cardiologists think that we do have a national guideline for fitness to drive, although this is not the case. The Saudi Commission for Health Specialties implemented a Code of Ethics for Healthcare Practitioners, ${ }^{8}$ which includes their duties towards patients and the community. The duties involve educating patients

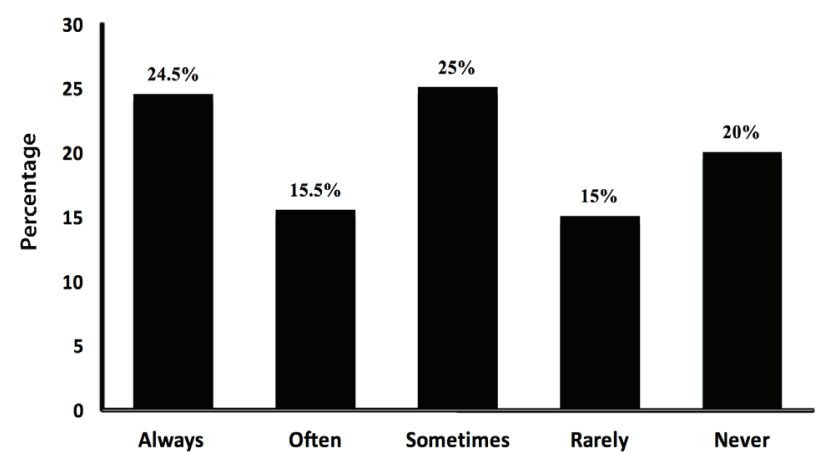

Figure 1 - The overall percentage of cardiologists' attitudes toward assessing medical fitness to drive in different cardiovascular diseases. about their particular diseases, and how to maintain their health through suitable means of protection that include proper advice and precautions. Furthermore, it includes an endorsement of all possible measures to protect the environment. Unfortunately, there were no specific national Saudi medical driving regulations; however, the ministry of health has provided free online access to UpToDATE ${ }^{\circledR 9}$ for health practitioners in KSA, which includes a separate chapter on the evaluation of older drivers and their fitness. Importantly, there is a huge debate worldwide about the legal and ethical responsibilities of whether assessment and advice regarding medical fitness to drive should be undertaken either by family physicians or specialists. ${ }^{10}$ However, CVD incapacitation assessment requires an expert cardiologist mainly when dealing with implanted devices such as pacemakers, implantable cadioverter defibrillators (ICDs), and cardiac assist devices. ${ }^{11}$

Few studies have been conducted to assess the awareness of healthcare workers in KSA. Alkharboush et $\mathrm{al}^{6}$ surveyed primary care physicians, and concluded that only $15 \%$ of the study participants considered themselves responsible for reporting driving fitness, while the rest mistakenly believed that this was solely the duty of either the patients themselves or the local traffic authorities.

It seems highly essential to divide the CVD into separate chapters when assessing fitness to drive and update them frequently. ${ }^{2}$ Cardiovascular diseases vary significantly in symptoms and disease severity, which may result in variable decisions for different disorders. ${ }^{4,8,12}$ Importantly, syncope is the most common CVD symptom preceding a motor vehicle accident, thus, it is vitally important to consider the risk of harm in patients with CVD who are at high risk of fainting. In addition, a lower threshold and longer periods of driving bans should be applied when dealing with patients who work as drivers of trucks or large vehicles in public or commercial transportation, which may result in an increased risk to the general public. ${ }^{13}$

Globally, driving regulations differ widely among nations; in addition, there is variability among studies and guidelines in regard to fitness-to-drive and the restriction periods in CVD and all other diseases. This make the physician's decision more subjective depending on the final diagnosis, disability, disease longevity, and the type of license issued, whether commercial or noncommercial driving class. ${ }^{2,11,14}$ In 2016, the German guidelines added a new chapter particularly for CVD. This chapter included ICDs, syncope, coronary artery diseases, heart failure, cardiomyopathies, valvular diseases, and hypertension as a separate category. ${ }^{14}$ 
Likewise, the Canadian Cardiovascular Society $(\mathrm{CCS})^{11}$ updated their guidelines between 2003 and 2012 in regard to left ventricular assisted devices. ${ }^{11,15}$ The older recommendation was permanently banning driving activity for patients with the pulsatile models of left ventricle assisted devices because of their limited durability and high associated mortality. However, the CCS has amended the guidelines to allow patients with continuous-flow devices to drive if they have remained stable for 2 months' post device implementation. In addition, the CCS developed a quantitative approach using a formula to estimate the risk of harm called the CCS Fitness to Drive Risk of Harm Formula, which is as follows: TD $\times \mathrm{SCI} \times \mathrm{V} \times \mathrm{AC}$, where TD stands for fractional time spent driving, SCI is the likelihood of syncope, $\mathrm{V}$ is the type of vehicle, and $\mathrm{AC}$ is the probability that a syncopal spell during driving will result in a fatal or injury-producing accident. ${ }^{15}$

Our study has important clinical implications by shedding light on cardiologists' lacking and inconsistent knowledge regarding indications and the period of driving cessation in patients with symptomatic CVD; thus, awareness campaigns and careful education might potentially be required to improve knowledge among cardiologists in particular. Furthermore, the findings highlight the emergent need for formulation of as well as legislation concerning appropriate national regulations on driving fitness through effective communication between the concerned agencies including the Ministry of Health, the National Traffic Authorities, and the Saudi Heart Association.

The study limitations included the enrolled cardiologists and mainly focused on the most common types of CVD. Thus, larger studies that include different medical specialties and a wider range of medical disorders are needed.

In conclusion, awareness regarding driving fitness among cardiologists with various levels of experience needs to be improved in Saudi Arabia. Formulation of and legislation concerning medical driving fitness regulations, as well as a further education, may play important roles in improving awareness among health practitioners in Saudi Arabia.

Received 2nd September 2018. Accepted 27th November 2018.

From the Department of Cardiology (Abazid, Eldesoky, Alharbi, Smettei), Prince Sultan Cardiac Center Qassim, King Fahad Specialist Hospital, Department of Pharmacology (Almeman), Department of Medicine, College of Medicine, Qassim University, Buraydah, Kingdom of Saudi Arabia Department of Cardiology (Sakr), King Saud Complex, Department of Cardiology (Eissa), Hail Cardiac Center, Riyadh, Kingdom of Saudi Arabia.

Address correspondence and reprints request to: Dr. Rami Abazid, Department of Cardiology, PrinceSultan Cardiac Center-Qassim, King FahadSpecialist Hospital, Qassim, Buraydah, Kingdom of Saudi Arabia.E-mail: ramiabazid@yahoo.com ORCID ID: orcid.org/0000-0002-8504-1930

\section{References}

1. Mansuri FA, Al-Zalabani AH, Zalat MM, Qabshawi RI. Road safety and road traffic accidents in Saudi Arabia. A systematic review of existing evidence. Saudi Med J 2015; 36: 418-424.

2. Driver and Vehicle License Agency. Assessing Fitness to drive: a guide for medical professionals. Updated: 2018. Accessed from: https://www.gov.uk/government/publications/assessing-fitnessto-drive-a-guide-for-medical-professionals

3. Numé AK, Gislason G, Christiansen CB, Zahir D, Hlatky MA, Torp-Pedersen C, et al. Syncope and motor vehicle crash risk: a danish nationwide study. JAMA Intern Med 2016; 176: 503-510.

4. Marino M, de Belvis A, Basso D, Avolio M, Pelone F, Tanzariello $\mathrm{M}$, et al. Interventions to evaluate fitness to drive among people with chronic conditions: systematic review of literature. Accid Anal Prev 2013; 50: 377-396.

5. Simpson C, Ross D, Dorian P, Essebag V, Gupta A, Hamilton R, et al. CCS consensus conference 2003: assessment of the cardiac patient for fitness to drive and fly-executive summary. Can J Cardiol 2004; 20: 1313-1323.

6. Alkharboush GA, Al Rashed FA, Saleem AH, Alnajashi IS, Almeneessier AS, Olaish AH, et al. Assessment of patients' medical fitness to drive by primary care physicians: A crosssectional study. Traffic Inj Prev 2017; 18: 488-492.

7. Simpson CS, Hoffmaster B, Mitchell LB, Klein GJ. Mandatory physician reporting of drivers with cardiac disease: ethical and practical considerations. Can J Cardiol 2004; 20: 1329-1334.

8. Saudi Commission for Health Specialties. In: Crisera V, editor. Code of Ethics for Healthcare Practitioners. Riyadh (KSA): Saudi Commission for Health Specialties; 2014.

9. Ladden MD. Approach to the evaluation of older drivers. UpToDate ${ }^{\circledR}$. Update: 2017. Accessed from: https://www.uptodate.com/contents/approach-to-theevaluation-of-older-drivers

10. Laycock KM. Should family physicians assess fitness to drive?: no. Can Fam Physician 2010; 56: 1265-1267.

11. Baskett R, Crowell R, Freed D, Giannetti N, Simpson CS; Canadian Cardiovascular Society. Canadian Cardiovascular Society focused position statement update on assessment of the cardiac patient for fitness to drive: fitness following left ventricular assist device implantation. Can J Cardiol 2012; 28: 137-140.

12. Banning AS, $\mathrm{Ng}$ GA. Driving and arrhythmia: a review of scientific basis for international guidelines. Eur Heart J2013; 34 : 236-244.

13. Tan VH, Ritchie D, Maxey C, Sheldon R, on behalf of the POST Investigators. Vasovagal syncope during driving: assessment using risk of harm formula and post-1 and post-2 studies. Canadian Journal of Cardiology 2013; 29: S380-S381.

14. Jung W, Hajredini B, Zvereva V. Fitness to drive with cardiovascular diseases: current guidelines of the German Federal Highway Research Institute. Herz 2018; 43: 367-380.

15. Simpson C, Dorian P, Gupta A, Hamilton S, Hoffmaster B, Klein B, et al. Assessment of the cardiac patient for fitness to drive: drive subgroup executive summary. Can J Cardiol 2004; 20: 1314-1320. 